\title{
Fuzzy Behavior Modulation with Threshold Activation for Autonomous Vehicle Navigation*
}

\author{
Edward Tunstel \\ Robotic Vehicles Group \\ Jet Propulsion Laboratory \\ California Institute of Technology \\ Pasadena, CA 91109
}

\begin{abstract}
This paper decribes fuzzy logic techniques used in a hierarchical behavior-based architecture for robot navigation. An architectural feature for threshold activation of fuzzybehaviors is emphasized, which is potentially useful for tuning navigation performance in real world applications. The target application is autonomous local navigation of a small planetary rover. Threshold activation of low-level navigation behaviors is the primary focus. A preliminary assessment of its impact on local navigation performance is provided based on computer simulations.
\end{abstract}

\section{Introduction}

Recent literature has reported numerous applications of fuzzy logic to challenging problems in autonomous control of electromechanical systems. In many cases, researchers have found it beneficial to address the challenges by approaching the control problem using multiple special-purpose fuzzy controllers, each responsible for a relatively simple, but integral, aspect of the problem. A common issue associated with such implementations is the determination of a suitable mechanism for selecting and/or coordinating the most appropriate controller(s) for a given system context. In addition, it is desirable to embed some capacity for tuning the behavior of the system when it is applied in real world operations.

This paper describes an architecture for autonomous control that employs multiple fuzzy controllers. The architecture has proven useful on mobile robots for realizing behavior-based local navigation, i.e. sensorbased navigation without the use of a global map. As designed, each controller implements a fuzzy logicbased motion control behavior or decision-making behavior that is integral to the navigation task. A

- The research described in this publication was carried out by the Jet Propulsion Laboratory, California Institute of Technology, under contract with the National Aeronautics and Space Administration. fuzzy-behavior selection and coordination mechanism called behavior modulation already exists in the architecture. It is based on a weighted decision-making scheme that involves scaling and aggregation of fuzzy sets, which represent outputs of behaviors that are applicable in the current context. In prior research based on this fuzzy control architecture, threshold activation of behaviors was not exploited. This paper explores this additional architectural degree-of-freedom for potential impacts on local navigation performance. It is intended as a preliminary investigation of beneficial effects derived from threshold-based execution of fuzzy-behaviors. The fuzzy behavior-based controller is briefly described, followed by a proposed strategy for behavior activation using fixed thresholds. The proposed approach is then applied to autonomous local navigation of a small planetary rover. The effects of threshold activation are demonstrated via comparative behavioral responses of the rover in computer simulations. Finally, conclusions are stated.

\section{Rule-based Behavior Hierarchy}

The functionality required for autonomous navigation behavior can be distributed across a finite number of special-purpose task-achieving behaviors. An effective arrangement of behaviors as a hierarchy of distributed fuzzy rule-bases was recently proposed for autonomous navigation in unstructured environments [1]. A collection of primitive behaviors residing at the lowest level are encoded as fuzzy rule-bases with distinct control policies governed by fuzzy inference. They are typically simple and self-contained behaviors that serve a single purpose while operating in a reactive or reflexive fashion. Examples include simple hazard avoidance and motion towards commanded sub-goals. Primitive behaviors perform mappings from different subsets of the sensor suite to common actuators. They also function as building blocks for higher-level coor- 
dination behaviors, referred to as composite behaviors, such as goal-seeking or route-following [1]. Hereafter, references to primitive and composite fuzzy-behaviors will be abbreviated as p-behaviors and c-behaviors, respectively.

Let $X$ and $U$ be input and output universes of discourse of a p-behavior with a rule-base of size $N$. We describe the generic fuzzy if-then rule as follows

$$
\text { IF } x \text { is } \tilde{A}_{i} \text { THEN } u \text { is } \tilde{B}_{i}
$$

where $x$ and $u$ represent input and output fuzzy linguistic variables, respectively, and $\tilde{A}_{i}$ and $\tilde{B}_{i}(i=$ $1,2, \ldots, N)$ are fuzzy subsets representing linguistic values of $x$ and $u$. In the rover controller, $x$ refers to sensory data or goal information; $u$ refers to setpoints for motor control velocities. P-behaviors behaviors consist of a finite set of such rules. Formally, the output of the $i$-th fuzzy rule is represented by a fuzzy relation, $\tilde{u}_{i} \in X \times U$, which is a fuzzy set itself. Moreover, the output of a fuzzy rule-base can be characterized as a single fuzzy relation, $\tilde{\beta}$, which is a union of fuzzy relations $\tilde{u}_{i}, i=1,2, \ldots, N$. Outputs of a $p-$ behavior, then, can also be represented as fuzzy sets. In our application they are fuzzy sets of recommended wheel velocities for the motors to achieve. Alternatively, they could represent vehicle steering and speed.

\subsection{Fuzzy-behavior modulation}

One way to coordinate, or resolve conflicts between, several recommendations is to aggregate them via fuzzy union and defuzzify the result to yield a crisp control action. However, in many cases, such fusion of recommendations does not provide sufficient decisionmaking flexibility for autonomous control. What is needed is a mechanism for controlling the amount of influence a particular behavior has on the control action in a context-dependent way. The architecture provides this flexibility by incorporating a scheme called behavior modulation, which we define as the continuous adjustment or adaptation of behavior activation levels in a multi-behavior or multi-agent system [1]. Behavior modulation is achieved by weighted decisionmaking, which is regulated through assignment of activation levels to each p-behavior. Activation levels govern the extent to which each behavior is applicable in the current context as perceived from goal information, sensory input, and internal state. The rover's composite behaviors serve as meta-rule-bases in which individual fuzzy rules have weighting consequents that specify the instantaneous activation level of $p$-behaviors. Hereafter, rules that modulate the activity of $\mathrm{p}$-behaviors in this way will be referred to as activation rules. The activation level, $\alpha$, of a p-behavior is specified in the consequent of activation rules of the form

$$
\text { IF } x \text { is } \tilde{A}_{i} \text { THEN } \alpha \text { is } \tilde{D}_{i}
$$

where $\tilde{A}_{i}$ is defined as in (1). $\tilde{D}_{i}$ is a fuzzy subset representing the linguistic value (e.g. high, low, etc) of the behavior's activation level to the situation prevailing during the current control cycle. It is defined over the closed unit interval, $[0,1]$. In general, a c-behavior, $c$, will include activation rules such as (2) for each $\mathrm{p}$ behavior $p$ modulated by $c$. Thus, for all $p, \alpha_{p} \in[0,1]$ is determined by fuzzy inference as the output of an associated c-behavior.

Let us denote the fuzzy output of primitive behavior $p$ as $\tilde{\beta}_{p}$, and its corresponding activation level as $\alpha_{p}$. Let $P$ be the set of all p-behaviors in a given behavior hierarchy. Then the modulated fuzzy output of $p$ is given by $\tilde{\beta}_{p}^{M}=\left(\alpha_{p} \cdot \tilde{\beta}_{p}\right)$. Modulated fuzzy outputs for all $p$ are aggregated using an appropriate t-conorm to produce the resultant output of the behavior hierarchy. The arithmetic sum t-conorm has been chosen for this purpose since it facilitates enforcement of the weighted decision-making intended in the philosophy the proposed approach. The arithmetic sum will be denoted by the symbol, $\uplus$. Finally, if we denote the output fuzzy set of the behavior hierarchy as $\tilde{\beta}_{H}$, then it can be computed as follows

$$
\tilde{\beta}_{H}=\biguplus_{p \in P} \alpha_{p} \cdot \tilde{\beta}_{p} .
$$

The crisp control output, $u^{*} \in U$, which serves as the velocity set-point input for the wheel motors, is computed by the Center-of-Sums defuzzification [2] of $\tilde{\beta}_{H}$.

$$
u^{*}=\frac{\int_{u \in U} u \sum_{p \in P} \alpha_{p} \cdot \mu_{\bar{\beta}_{p}}(u)}{\int_{u \in U} \sum_{p \in P} \alpha_{p} \cdot \mu_{\dot{\beta}_{p}}(u)}
$$

This expression is the nonlinear input-output mapping of the fuzzy-behavior hierarchy which adapts dynamically due to continuous fluctuations in $\alpha_{p}, \forall p \in P$. In this procedure, multiplication by $\alpha_{p}$ expresses the relative applicability of a p-behavior to the current situation, while the scalar $\alpha_{p}$ itself represents the weight of the behavior in the aggregated control decision. Operators other than multiplication can be used to achieve a similar effect. Yager [3] refers to such operators as importance transformations and suggests a general class of them for both t-norm and t-conorm aggregations.

This coordination method is attractive because it allows robots to exhibit controlled behavioral responses throughout a continuum between cooperation and competition. In any control cycle, resultant control action is a consensus of controls recommended by all 
applicable behaviors. This is in contrast to non-fuzzy behavior arbitration schemes, which typically employ fixed priorities that allow only one active behavior during a given control cycle. The behavioral consensus of control facilitates smooth execution of motion commands. This strategy is particularly suitable in the context of fuzzy-behavior hierarchies.

\section{Threshold Activation}

In a single fuzzy rule-base, the firing of a rule produces a fuzzy set derived from the rule consequent, the size of which is related to the firing strength of the rule. The resulting output fuzzy set of the rule can be viewed as a measure of stimulus induced by the rule inputs. In recent work, Maeda et al [4] propose an effective modification of Zadeh's fuzzy algorithm [5] in which the firing of individual rules is governed by adjustable thresholds. In systems comprised of multiple fuzzy controllers, this concept can also be effectively applied at the coarser granularity of rulebases. This was alluded to in [5] as a possible mode of execution used by humans to execute a set of fuzzy instructions; there, it is referred to as nondeterministic execution with threshold. For behavior-based systems, this translates into a means for tuning individual systems of multiple behaviors. The thresholds imposed on behavior activation allow filtering of undesirable inter-behavioral influences that might be observed during system testing or operation. As a practical utility, thresholds are the tunable "knobs" of the system that permit performance refinement in different operating environments. Such flexibility is desirable for real world applications of mobile robots since the performance of pre-programmed behavior can vary from environment to environment (e.g. sparsely cluttered to densely cluttered domains). This was attested to in a recent analysis of variations in expected navigation performance of rovers based on different obstacle distributions and vehicle size [6]. Thus far, research proposing similar fuzzy coordination strategies as that described above has not emphasized the utility of thresholds in this manner.

Herein, we attempt to elevate the notion of stimulus from the rule level to the level of rule-bases. Like fuzzy rules, fuzzy rule-bases (behaviors in our case) also produce an output fuzzy set, which results from fuzzy inference. In an analogous manner, the output fuzzy set of a behavior can be viewed as a stimulus induced by the behavior input(s). In general, the stronger the stimulus, the more responsive the behavior. However, the hierarchy determines the ultimate activation level of the behavior based on its current applicability as described above. The modulated fuzzy output that results is a measure of the behavior's stimulus from the point of view of the hierarchy. It is this stimulus that we impose activation thresholds upon for each p-behavior. Activation strategies can be formulated based on the consideration of the $\alpha$-cuts of behavior output fuzzy sets relative to fixed activation thresholds. Note that to avoid confusion with our use of the symbol $\alpha$ to designate activation levels, we will refer to the $\alpha$-cuts of fuzzy set theory as $\theta$-cuts herein. A $\theta$-cut of a fuzzy set, $\tilde{C}$, defined over a universe $U$, is the crisp set $\tilde{C}_{\theta}$ defined as

$$
\tilde{C}_{\theta}=\left\{x \in X \mid \mu_{\tilde{C}}(x) \geq \theta\right\}
$$

where $\mu_{\tilde{C}}(\cdot): X \rightarrow[0,1]$ is a membership function defining $\tilde{C}$. To utilize $\theta$-cuts for implementing threshold behavior activation, we consider the modulated fuzzy output of the behavior, $\tilde{\beta}_{p}^{M}$. If the $\theta$-cut of the modulated fuzzy output is null, then the system recommends that the level of activation for the associated behavior is zero. That is, if a threshold $\theta \in[0,1]$ is imposed on a behavior $p$, its modulated fuzzy output is augmented to determine its activation according to

$$
\tilde{\beta}_{p}^{M}=\left\{\begin{array}{rll}
\tilde{\theta} & : & \left(\tilde{\beta}_{p}^{M}\right)_{\theta}=\emptyset \\
\tilde{\beta}_{p}^{M} & : & \left(\tilde{\beta}_{p}^{M}\right)_{\theta} \neq \emptyset
\end{array}\right.
$$

where $\tilde{\emptyset}$ denotes the null fuzzy set. In the interest of computational efficiency, the implementation avoids unnecessarily computing the modulated fuzzy set to determine its $\theta$-cut. Instead, we compare its height to the threshold so that the behavior is activated if $\alpha_{p} \cdot \max \left(\mu_{\tilde{\beta}_{p}^{M}}\right) \geq \theta$. The operational premise, then, is that behaviors are activated if their stimuli exceed their activation thresholds. Behavior selection is a special case of this approach that occurs when the stimulus of a p-behavior exceeds its activation threshold while the stimuli of other p-behaviors is zero or below threshold.

\section{Rover Local Navigation}

This preliminary investigation of threshold activation is ultimately geared towards applications to planetary rover navigation. Autonomous rovers designed for planetary surface exploration must be capable, at least, of point-to-point local navigation in the presence of varying obstacle distributions, surface characteristics, and hazards. Often the navigation task is facilitated by awareness of a series of waypoints (sub-goals), furnished by human operators or a path planner, which lead to designated goals. In some cases, control must be achieved without the luxury of continuous or frequent remote communication between an Earth-based mission control station and the rover. Rover autonomy 


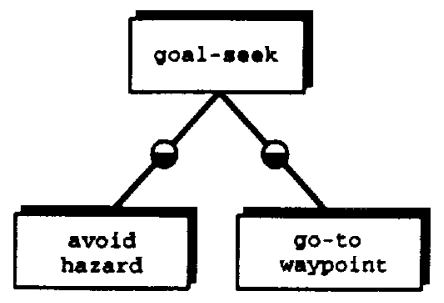

Figure 1: Behavior hierarchy for local navigation.

capabilities must be provided under significant constraints on power, computation, weight, and communications. To further increase the challenge, many popular state-of-the-art processors that enable advanced capabilities in laboratory research robots is infeasible. This is due to the fact that space flight projects require the use of proven, radiation-hardened, or otherwise space flight-qualified electronics. The meager availability of fast and/or powerful space-qualified processors for on-board computation fuels the need for efficient algorithms for implementing the necessary on-board autonomy. Considering these and other constraints associated with rover navigation, a fuzzy-behavior hierarchy for planetary rovers was suggested in [1]. A portion of that hierarchy with sufficient functionality for local navigation is shown in Fig. 1. In this figure the behavioral function of goal-seek (collisionfree navigation to a goal location) is decomposed into the two p-behaviors shown. The interconnecting circles between the levels of hierarchy represent weights and activation thresholds associated with p-behaviors. As its name implies, the purpose of the avoid-hazard behavior is to avoid hazards such as large rocks. The go-to-waypoint behavior will direct the rover to traverse a straight line trajectory to a specified waypoint. The goal-seek behavior coordinates these using behavior modulation with threshold activation.

As the target system for implementation of the behavior hierarchy, we consider a class of miniature rovers being developed for planetary surface exploration missions. The largest dimension (length) of an existing prototype rover is $20 \mathrm{~cm}$, which makes it $30 \%$ the size of the NASA rover, Sojourner, deployed on Mars in July of 1997. The utility of such rovers for in-situ surface exploration as individual units or cooperative teams is currently being investigated [6]. The vehicle under consideration is driven by a four-wheel mobility chassis that is differentially steered. Below, we demonstrate the effect of threshold activation on a simulated model of this rover. The primary navigation sensing capabilities considered here include wheel odometry and obstacle range sensing.

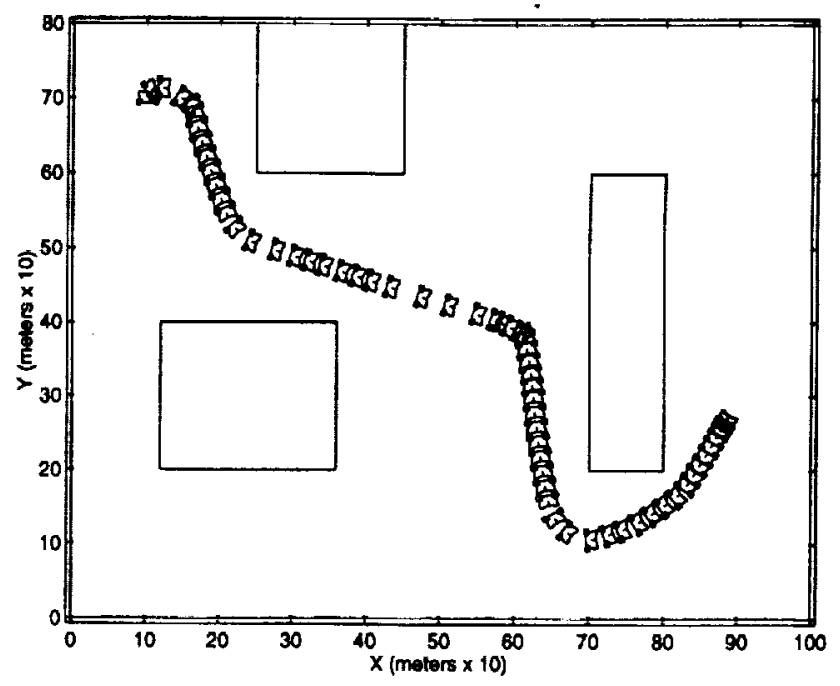

Figure 2: Successful path without thresholds.

\subsection{Preliminary result}

Two cases are considered, namely, nominal behavior hierarchy design $\left(\theta_{p}=0 \forall p\right)$ and threshold activation. Performance is predicted by simulated navigation in two distinct environments with the mere intent of drawing attention to performance variations that could occur from environment to environment. For the sake of demonstrating potential effects of threshold activation, the simulations are kept simple and are implemented with relatively low fidelity. The first environment is a "block world" of several obstacles. It represents an environment wherein the local navigator performs quite well. The second environment is a field cluttered with randomly distributed "rocks" of various sizes that are considered obstacles/hazards for the rover. In both cases, the terrain is assumed to be twodimensional. This is an over-simplification of actual rover mission scenarios in which complex motions in the third dimension occur quite frequently. However, this assumption still allows examination of obstacle negotiating capabilities of the local navigator.

We have simulated the obstacle range sensor coverage area to be limited to $\approx 3 m$ spanning a frontal field of view of $\approx 2.5 \mathrm{rad}(145 \mathrm{deg})$. A successful goal-seeking result in the first environment is shown in Fig. 2 where the task is to navigate from initial pose $(x y \phi)=\left(1 \mathrm{~m} 7 \mathrm{~m} \frac{\pi}{2} \mathrm{rad}\right)$ to a goal at location $(9 m 3 m)^{1}$. The controller is executed in its nominal configuration with zero thresholds for p-behaviors in Fig. $1\left(\theta_{a h}=\theta_{g w}=0\right)$. This result is representative of point-to-point navigation performance in this

\footnotetext{
${ }^{1}$ Goal orientation is not specified; it is assumed that simply rotating in place will satisfy such requirements.
} 


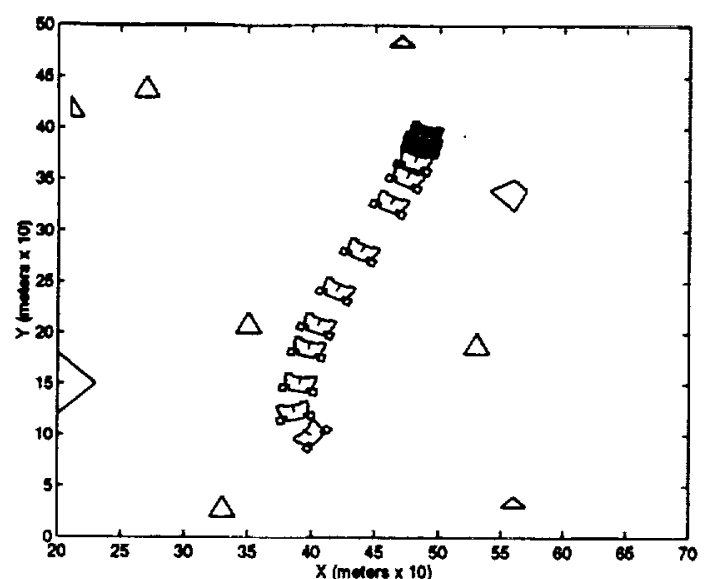

Figure 3: Failed path without thresholds.

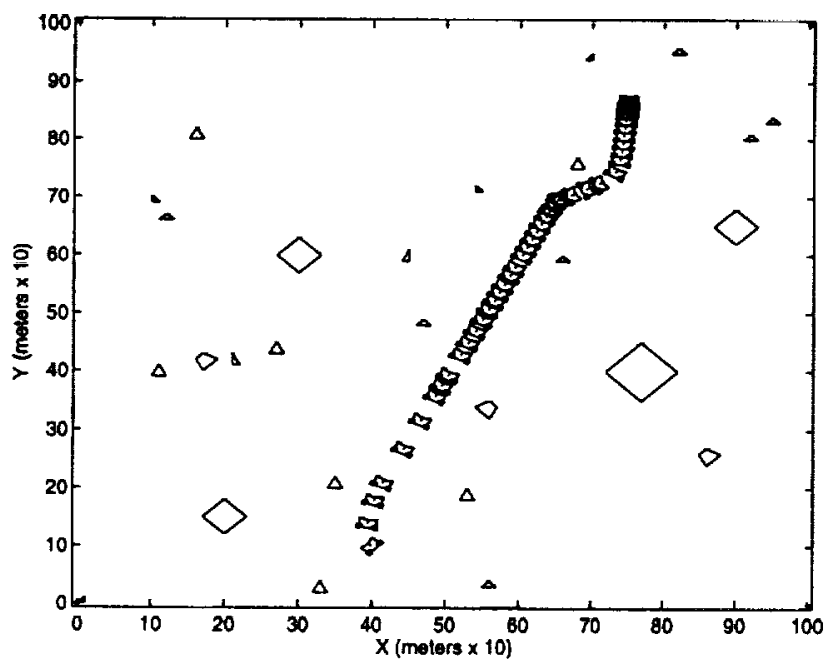

Figure 4: Successful path with threshold activation.

environment for many initial states and goals. When the vehicle is deployed in the rock field, using the same navigation controller, it exhibits a degradation in performance for similar tasks. Fig. 3 shows a portion of the field in which a failure occurs. In the rock field, the task is to navigate from ( $4 \mathrm{~m} 1 \mathrm{~m} \mathrm{2.44rad)}$ to $(7.5 \mathrm{~m} 8.5 \mathrm{~m})$. With zero threshold settings the nominal controller reaches a deadlock near point $(5 \mathrm{~m} 4 \mathrm{~m})$ and fails advance further towards the goal.

Thresholds of $\theta_{a h}=0.5$ and $\theta_{g w}=0.0025$ were then applied to govern activation of the p-behaviors for the case in Fig. 3. For this example, these values were determined based on a sparse, but uniform, trial-anderror sampling of the space of thresholds. The successful path traversed using these thresholds is shown in Fig. 4. In this case, threshold activation enables a consensus to be reached that does not result in fail- ures such as deadlocks or similar artifacts of behavior interaction. Based on these preliminary results, it is expected that threshold activation would be useful for adjusting performance characteristics of systems with even more behaviors than used in our example.

\section{Conclusions}

Application of thresholds effects behavior-based controllers by altering nominal modes of interaction between p-behaviors. Thresholds have the effect of filtering inter-behavioral influences that cause success in one environment and performance degradation in others. Threshold activation appears useful as a means of tuning system behavior for compliance with different environments with a minimum of controller re-design. In lieu of applied thresholds, performance refinement can be achieved effectively with a thorough effort of tuning membership functions, adjusting scaling factors, and/or modifying rules. Where feasible, thresholds may be the simpler approach depending upon the level of effort required. However, thresholds for behavior activation can be difficult to choose. The difficulty increases with the addition of behaviors to the system, in which case parameter learning algorithms may be the better option. In fact, on-line learning is a desirable feature for robotic vehicles that may be deployed in varied operating domains. However, they can be difficult to apply successfully, particularly on resourceconstrained systems such as planetary rovers. Threshold activation is an acceptable alternative to the more ambitious provision of an on-line learning capability.

\section{References}

[1] E. Tunstel, T. Lippincott, and M. Jamshidi. Behavior hierarchy for autonomous mobile robots: Fuzzybehavior modulation and evolution. Intl Jrnl of Intelligent Automation \& Soft Computing, 3(1):37-49, 1997.

[2] D. Driankov, H. Hellendoorn, and M. Reinfrank. An Introduction to Fuzzy Control. Springer-Verlag, Berlin, Germany, 1993.

[3] R.R. Yager and D.P. Filev. Essentials of Fuzzy Modeling and Control. Wiley and Sons, New York, 1994.

[4] Y. Maeda et al. Hierarchical control for autonomous mobile robots with behavior-decision fuzzy algorithm. In IEEE Intl Conf on Robotics \& Automation, pages 117-122, Nice, France, May 1992.

[5] L. A. Zadeh. Fuzzy algorithms. Information and Control, 12:94-102, 1968.

[6] B. Wilcox, A. Nasif, and R. Welch. Implications of Martian rock distributions on rover scaling. In /st Intl Conf on Mobile Planetary Robots, Santa Monica, CA, Feb 1997. 